\title{
Optical Interference Signal Processing in Precision Dimension Measurement
}

\author{
Haijiang $\mathrm{Hu}^{1}{ }^{,}$, , Fengdeng Zhang ${ }^{2}$, Juju $\mathrm{Hu}^{1}$ and Yinghua $\mathrm{Ji}^{1}$ \\ 1Jiangxi Normal University, Nanchang, \\ ${ }^{2}$ University of Shanghai for Science and Technology, Shanghai,
}

China

\section{Introduction}

The optical techniques are widely used in the automatic measurement and test. In the precision dimension measurement, there are some optical instruments that are widely applied for the microscale or nanoscale dimension measurement, such as the grating, the homodyne interferometer, and the heterodyne interferometer. An optical instrument system is often divided into two main subsystems: the optical subsystem and the electrical subsystem. Moreover, some optical instrument systems also have the mechanical subsystem and the computer subsystems. The optical subsystem includes optical source and other optical elements, such as the polarizing beam splitter, the quarter-wave plate, and so on. The electronic subsystem turns the received optical signals into the electrical signals by the photoelectric receiver, and uses the analog signal system and the digital signal system to process the received signals. A typical structure of the optical instruments is shown as Fig.1.

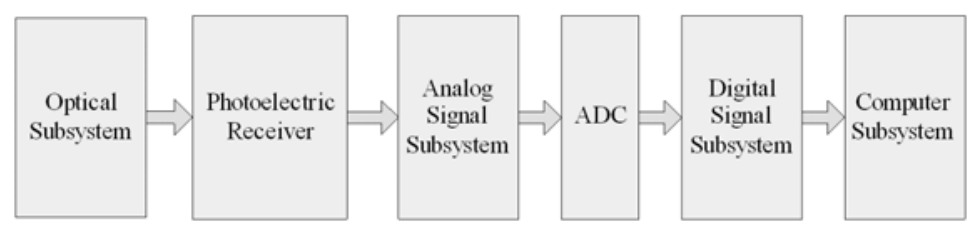

Fig. 1. A typical structure of optical instruments: ADC, analog-digital converter.

In the performance evaluation of the optical measurement instrument, the resolution and the precision (JCGM, 2008) are two important parameters. The resolution describes the ability of a measurement system to resolve detail in the object that is measured. The precision of a measurement system, also called reproducibility or repeatability, is the degree to show the same results when the measurement process is repeated under the unchanged conditions. This chapter mainly discusses two topics: the signal processing method for the improvement of measurement resolution and the elimination errors for the improvement of measurement precision. Generally speaking, the optical source, the design of optical path, and the signal processing are the main factors to improve the measurement resolution. For

${ }^{*}$ Corresponding Author 
example, in a heterodyne Michelson interferometer with double path optical difference that is shown as Fig.2, the traveling distance of the micro-displacement platform $L$ is as follows:

$$
L=\frac{\lambda}{2} \int_{0}^{T} \Delta f d t
$$

where $\lambda$ is the wavelength of laser, and $\Delta f$ is the Doppler frequency shift. Obviously, $\lambda$ is smaller, the measurement resolution is higher.

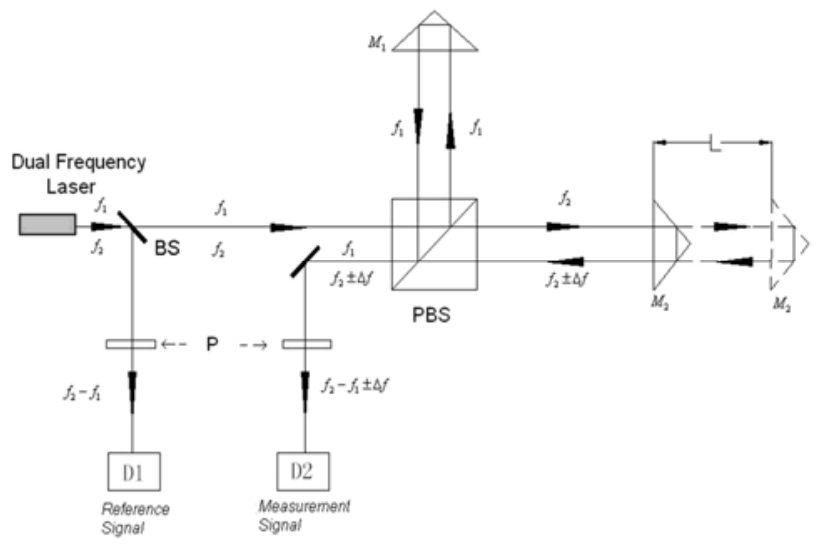

Fig. 2. A heterodyne Michelson interferometer with double path optical difference: BS, beam splitter; PBS, polarizing beam splitter; $\mathrm{P}$, polarization analyzer.

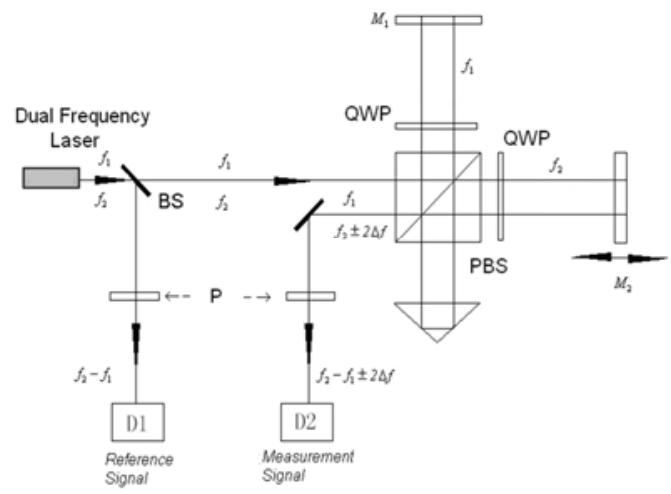

Fig. 3. A heterodyne Michelson interferometer with fourfold path optical difference: BS, beam splitter; PBS, polarizing beam splitter; $\mathrm{P}$, polarization analyzer; QWP, quarter-wave plate.

The multiple reflection of optical path, also called the optical subdivision, is the second factor to influence the measurement resolution. A heterodyne Michelson interferometer 
with fourfold path optical difference is shown as Fig.3. When the micro-displacement platform $\mathrm{M}_{2}$ moves the same distance $L$ in Fig.2 and Fig.3, the Doppler frequency shift $\Delta f$ in Fig.3 is twice as large as $\Delta f$ in Fig.2. In the same configuration of laser source and electrical signal processing system, the measurement resolution in Fig.3 is twice as large as the measurement resolution in Fig.2. Therefore, the optical subdivision relies on the optical path design of optical system and can be realized by the multiple reflections in the optical path (Cheng et al., 2006).

The third method is the electrical signal processing. Because of the light attenuation in optical parts, the optical subdivision is hard to realize the high subdivision number. Moreover, the measurement error from the nonideal characters and arrangement of optical parts is gradually increased when the number of optical subdivision is added. So the electrical signal processing becomes the most useful method to improve the measurement resolution.

Moreover, the detection and elimination of errors is the important research targets for the improvement of measurement precision. These errors have very serious influence on the measurement precision. In this chapter we discuss electrical signal processing of orthogonal signals (fringes), which is widely used in the optical gratings, the homodyne interferometers, and other optical instruments.

\section{Signal preprocessing}

In the optical gratings and the homodyne interferometers, the photoelectric converter turns the intensity of light into the electrical signal. Because of the requirement of the direction recognition when the measured object moves, the orthogonal signals whose phase difference is $\pi / 2$ are used in these instruments. In order to obtain the orthogonal signals, 4channel signal receiving systems are designed in the optical systems. Fig.4 shows a typical 4channel signal receiving systems (Keem et al., 2004).

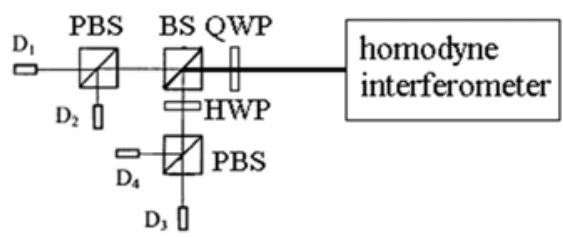

Fig. 4. 4-channel signal receiving systems: BS, beam splitter; PBS, polarizing beam splitter; QWP: quarter-wave plate; HWP: half-wave plate;

When the measured object moves forward, the 4-channel electrical signals are collected from the photosensors and shown as follows:

$$
\left\{\begin{array}{l}
I_{1}=A_{1}+B_{1} \sin \theta \\
I_{2}=A_{2}-B_{2} \cos \theta \\
I_{3}=A_{3}-B_{3} \sin \theta \\
I_{4}=A_{4}+B_{4} \cos \theta
\end{array},\right.
$$


where $A_{n}$ is the DC component of channel $n, B_{n}$ is the amplitude of AC component of channel $n$, and $\theta$ is the change of phase that is related to the displacement $\Delta L$ when the measured object moves, respectively. In the homodyne interferometer with two path optical differences, the relationship between $\theta$ and $\Delta L$ is shown as follows:

$$
\theta=\frac{4 \pi}{\lambda} \Delta L
$$

where $\lambda$ is the wavelength of laser.

Two signals $I_{x}$ and $I_{y}$ can be obtained by subtracting:

$$
\left\{\begin{array}{l}
I_{y}=I_{1}-I_{3} \\
I_{x}=I_{4}-I_{2}
\end{array}\right.
$$

From Eqs.2 and Eqs. 4,

$$
\left\{\begin{array}{l}
I_{y}=A_{1}-A_{3}+\left(B_{1}+B_{3}\right) \sin \theta \\
I_{x}=A_{4}-A_{2}+\left(B_{2}+B_{4}\right) \cos \theta
\end{array} .\right.
$$

Under the ideal condition, $A_{2}=A_{4}, A_{1}=A_{3}$ and $B_{2}+B_{4}=B_{1}+B_{3}$. So, two orthogonal signals are obtained as follows when the measured object moves forward:

$$
\left\{\begin{array}{l}
I_{y}=B \sin \theta \\
I_{x}=B \cos \theta^{\prime}
\end{array}\right.
$$

where $B=B_{1}+B_{3}=B_{2}+B_{4}$.

Similarly, the 4-channel signals are shown as follows when the measured object moves backward:

$$
\left\{\begin{array}{c}
I_{1}=A_{1}+B_{1} \sin \theta \\
I_{2}=A_{2}+B_{2} \cos \theta \\
I_{3}=A_{3}-B_{3} \sin \theta \\
I_{4}=A_{4}-B_{4} \cos \theta
\end{array}\right.
$$

where the parameters of Eqs.7 are the same as the parameters of Eqs.2. Similarly, from Eqs.4 and Eqs.7, two orthogonal signals are obtained as follows when the measured object moves backward:

$$
\left\{\begin{array}{c}
I_{y}=B \sin \theta \\
I_{x}=-B \cos \theta
\end{array},\right.
$$

where $B=B_{1}+B_{3}=B_{2}+B_{4}$.

So, $\Delta L$ is obtained by using the half periodic counting of orthogonal signals and the calculation of $\theta$ as follows: 


$$
\Delta L=\frac{\lambda}{2} N+\varepsilon,
$$

with

$$
\varepsilon=\frac{\Delta \theta}{4 \pi} \lambda,
$$

where $\lambda$ is the wavelength of laser, $N$ is the counting value of half period, and $\varepsilon$ is the additional displacement value that is shorter than $\lambda / 2$ and can be calculated from the orthogonal signals, respectively. For the improvement of measurement resolution, some bidirectional subdivision methods are studied to raise the resolution of $N$ and $\varepsilon$.

\section{Bidirectional subdivision}

\section{1 //4 bidirectional subdivision}

The simplest bidirectional subdivision method (Yoshizawa, 2005) is shown as Fig.5. This method is applied on both the analog orthogonal signals and the digital orthogonal signals. The principle of this method is shown in the right part in Fig.5. During the measured object moves forward, when signal $u_{2}$ crosses zero from positive to negative, signal $u_{1}$ is positive; when signal $u_{1}$ crosses zero from positive to negative, signal $u_{2}$ is negative; when signal $u_{2}$ crosses zero from negative to positive, signal $u_{1}$ is negative; when signal $u_{1}$ crosses zero from negative to positive, signal $u_{2 f}$ is positive. Therefore, the counting of four times in a period for forward movement is realized.

Similarly, during the measured object moves backward, when signal $u_{2}$ crosses zero from negative to positive, signal $u_{1}$ is positive; when signal $u_{1}$ crosses zero from positive to negative, signal $u_{2}$ is positive; when signal $u_{2}$ crosses zero from positive to negative, signal $u_{1}$ is negative; when signal $u_{1}$ crosses zero from negative to positive, signal $u_{2}$ is negative. From the above detection, the counting of four times in a period for bidirectional movement is realized.

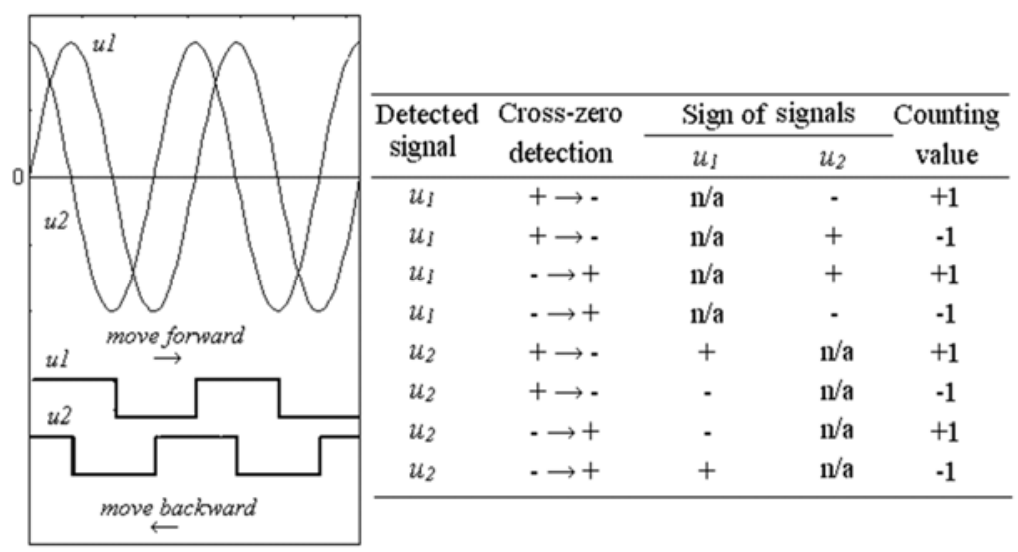

Fig. 5. $\lambda / 4$ bidirectional subdivision 


\section{2 ג/8 bidirectional subdivision}

In order to realize the counting of more than four times in a period, some methods are studied. The first method uses two thresholds to realize $\lambda / 8$ bidirectional subdivision by (Chu et al., 2003). The method defines two thresholds $T_{h}$ and $T_{l} . T_{h}$ is the amplitude of normalized signal in the $\pi / 4$ phase while $T_{l}$ is the negative of $T_{h}$. When $u_{1}$ cross across $T_{h}$ from the bottom to the top, the movement direction is positive if $u_{2}$ is positive at the same time or negative if $u_{2}$ is negative at the same time. Similarly, when $u_{1}$ cross across $T_{l}$ from the bottom to the top, the movement direction is positive if $u_{2}$ is positive at the same time or negative if $u_{2}$ is negative at the same time. By combination between $\lambda / 4$ bidirectional subdivision and two thresholds detection, the $\lambda / 8$ bidirectional subdivision is realized.

(Cui et al., 2000) proposes a method to realize $\lambda / 8$ bidirectional subdivision by the constructed function. The constructed function is as follows:

$$
f=\left|u_{1}\right|-\left|u_{2}\right|
$$

Fig. 6 shows the function images of $u_{1}, u_{2}$ and $f$. According to the change of sign of $u_{1}, u_{2}$ and $f, \lambda / 8$ bidirectional subdivision is achieved. The principle of this method is as shown in Fig.7.

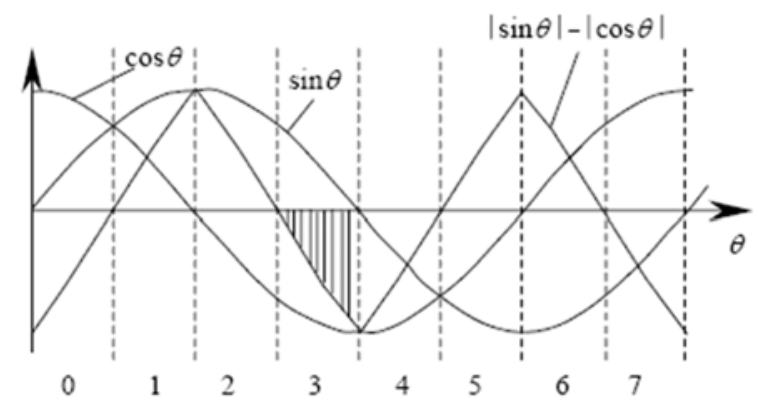

Fig. 6. Function images of $\mathrm{u}_{1}, \mathrm{u}_{2}$ and $\mathrm{f}$

\begin{tabular}{|c|c|c|c|c|c|}
\hline $\sin \theta$ & $\cos \theta$ & $f$ & code & status & \multirow{4}{*}{$\begin{array}{l}\text { move forward } \\
\mathrm{A} \rightarrow \mathrm{B} \rightarrow \mathrm{C} \rightarrow \mathrm{D} \rightarrow> \\
\mathrm{E} \rightarrow \mathrm{F} \rightarrow \mathrm{G} \rightarrow \mathrm{H} \rightarrow \mathrm{A}\end{array}$} \\
\hline$>0$ & $>0$ & $<0$ & 110 & A & \\
\hline$>0$ & $>0$ & $>0$ & 000 & B & \\
\hline$>0$ & $<0$ & $>0$ & 101 & C & \\
\hline$>0$ & $<0$ & $<0$ & 100 & D & move backward \\
\hline$<0$ & $<0$ & $<0$ & 000 & E & \multirow{4}{*}{$\begin{array}{l}\mathrm{A} \rightarrow \mathrm{H}->\mathrm{G}->\mathrm{F} \rightarrow \\
\mathrm{E} \rightarrow \mathrm{D} \rightarrow \mathrm{C}->\mathrm{B} \rightarrow \mathrm{A}\end{array}$} \\
\hline$<0$ & $<0$ & $>0$ & 001 & F & \\
\hline$<0$ & $>0$ & $>0$ & 011 & G & \\
\hline$<0$ & $>0$ & $<0$ & 010 & $\mathrm{H}$ & \\
\hline
\end{tabular}

Fig. 7. Principle of $\lambda / 8$ bidirectional subdivision by Eq.10 


\section{$3.3 \lambda / 16$ bidirectional subdivision}

Similar to (Cui et al., 2000), (Chen et al., 2005) proposes another constructed function and status code method to achieve $\lambda / 16$ bidirectional subdivision. The difference from (Cui et al., 2000), (Chen et al., 2005) uses 4 bits code as status parameter.

\section{$3.4 \lambda / 2^{n+2}$ bidirectional subdivision}

(Hu et al., 2009) and ( $\mathrm{Hu} \&$ Zhang, 2012) proposes a method of $\lambda / 2^{n+2}$ bidirectional subdivision that uses both the constructed function and the cross-zero detection. The $\lambda / 8$ and $\lambda / 16$ bidirectional subdivision methods are simply introduced at first. Commonly, two orthogonal signals are defined as follows when the measured object moves forward:

$$
\left\{\begin{array}{l}
u_{1 f}=U_{0} \sin \theta \\
u_{2 f}=U_{0} \cos \theta^{\prime}
\end{array}\right.
$$

where $U_{0}$ is the amplitude of the orthogonal signals, and $\theta$ is the change of phase that is related to the displacement when the measured object moves.

The following two relative functions are constructed to be the reference signals in order to achieve the bidirectional subdivision:

$$
\left\{\begin{array}{l}
r_{11 f}=U_{0} \sin \theta+U_{0} \cos \theta \\
r_{12 f}=U_{0} \sin \theta-U_{0} \cos \theta
\end{array} .\right.
$$

Reference signals $r_{11 f}$ and $r_{12 f}$ can be acquired by the digital adder. Therefore, the $\lambda / 8$ subdivision of the two orthogonal signals can be realized easily by the zero-cross detection which consists of the orthogonal signals and the reference signals.

Based on the $\lambda / 4$ bidirectional subdivision When the positive voltage drives the measured object to move forward, reference signal $r_{12 f}$ crosses zero from negative to positive, signals $\mathrm{u}_{1 f}$ and $\mathrm{u}_{2 f}$ are positive; when reference signal $r_{11 f}$ crosses zero from positive to negative, signal $\mathrm{u}_{1 f}$ is positive and signal $\mathrm{u}_{2 f}$ is negative; when reference signal $r_{12 f}$ crosses zero from positive to negative, signals $\mathrm{u}_{1 f}$ and $\mathrm{u}_{2 f}$ are negative; when reference signal $r_{11 f}$ crosses zero from negative to positive; signal $\mathrm{u}_{1 f}$ is negative and signal $\mathrm{u}_{2 f}$ is positive; Therefore, the counting of eight times in a period for the forward movement is realized.

The same to the forward counting, it's easy to realize the counting of eight times in a period for the backward movement. When the measured object moves backward, the two orthogonal signals are defined as follows:

$$
\left\{\begin{array}{c}
u_{1 b}=U_{0} \sin \theta \\
u_{2 b}=-U_{0} \cos \theta
\end{array} .\right.
$$

The same to Eqs.12, the two relative functions are constructed to be the reference signals:

$$
\left\{\begin{array}{l}
r_{11 b}=U_{0} \sin \theta-U_{0} \cos \theta \\
r_{12 b}=U_{0} \sin \theta+U_{0} \cos \theta
\end{array} .\right.
$$


Similarly, the counting of eight times in a period for the backward movement is also achieved. So, a set of reference signals are only built as follows:

$$
\left\{\begin{array}{l}
r_{11}=u_{1}+u_{2} \\
r_{12}=u_{1}-u_{2}
\end{array} .\right.
$$

The bidirectional subdivision of $\lambda / 8$ can be realized by the zero-cross detection of the orthogonal signals and the reference signals.

On the basis of the $\lambda / 8$ subdivision, a group of the reference signals is built which is constituted by the two sets of reference signals. During the time of forward counting, the reference signal sets are defined as follows:

$$
\begin{gathered}
\left\{\begin{array}{l}
r_{11 f}=U_{0} \sin \theta+U_{0} \cos \theta \\
r_{12 f}=U_{0} \sin \theta-U_{0} \cos \theta^{\prime}
\end{array}\right. \\
\left\{\begin{array}{l}
r_{21 f}=U_{0} \sin 2 \theta+U_{0} \cos 2 \theta \\
r_{22 f}=U_{0} \sin 2 \theta-U_{0} \cos 2 \theta
\end{array}\right.
\end{gathered}
$$

In order to calculate the reference signals $r_{21 f}$ and $r_{22 f}$, the two orthogonal signals are used to express them. $r_{21 f}$ is obtained as follows:

$$
r_{21 f}=U_{0} \sin 2 \theta+U_{0} \cos 2 \theta=U_{0}[2 \cos \theta(\sin \theta+\cos \theta)-1] .
$$

Similarly, $r_{22 f}$ is obtained as follows:

$$
r_{22 f}=U_{0}[2 \sin \theta(\sin \theta+\cos \theta)-1] .
$$

$U_{0}=1$ is still assumed. The function images of the two orthogonal signals and reference signals for the $\lambda / 16$ subdivision are shown in Fig. 8 and Fig. 9 during the time of the forward counting and the backward counting. The same as the $\lambda / 8$ subdivision, the crosszero detection is also used to realize the bidirectional subdivision of $\lambda / 16$. Therefore, on the basis of the $\lambda / 8$ subdivision, the bidirectional subdivision of $\lambda / 16$ for the orthogonal signals is realized by adding a set of reference signals:

$$
\left\{\begin{array}{l}
r_{21}=\frac{2 u_{2}}{U_{0}}\left(u_{1}+u_{2}\right)-U_{0} \\
r_{22}=\frac{2 u_{1}}{U_{0}}\left(u_{1}+u_{2}\right)-U_{0}
\end{array} .\right.
$$

Based on the $\lambda / 8$ subdivision and the $\lambda / 16$ subdivision, $n$ sets of reference signals can be built to realize the $\lambda / 2^{n+2}$ bidirectional subdivision as follows:

$$
\left\{\begin{array}{l}
r_{n 1}=U_{0} \sin 2^{n-1} \theta+U_{0} \cos 2^{n-1} \theta \\
r_{n 2}=U_{0} \sin 2^{n-1} \theta-U_{0} \cos 2^{n-1} \theta
\end{array},\right.
$$


where $n$ is integer and $n>0$.

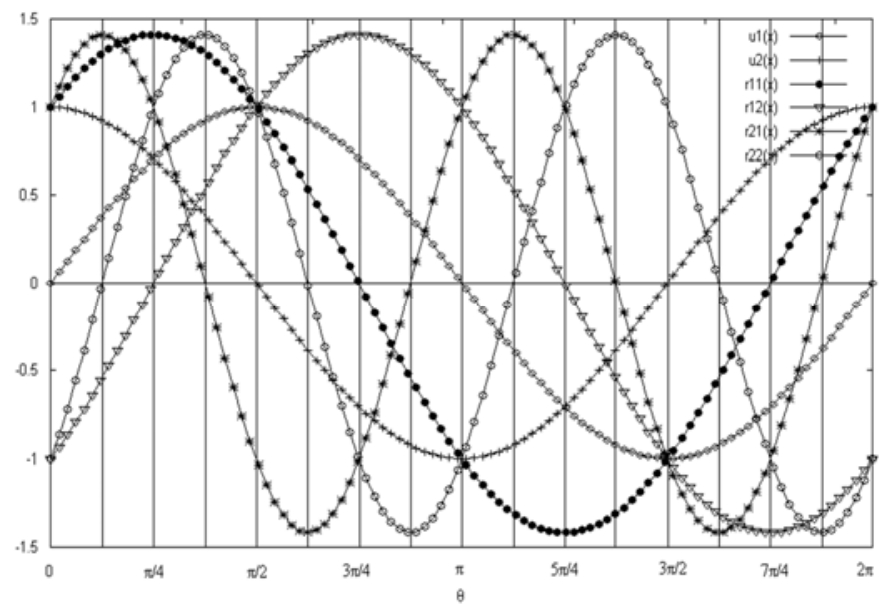

Fig. 8. Forward orthogonal signals and reference signals for $\lambda / 16$ subdivision

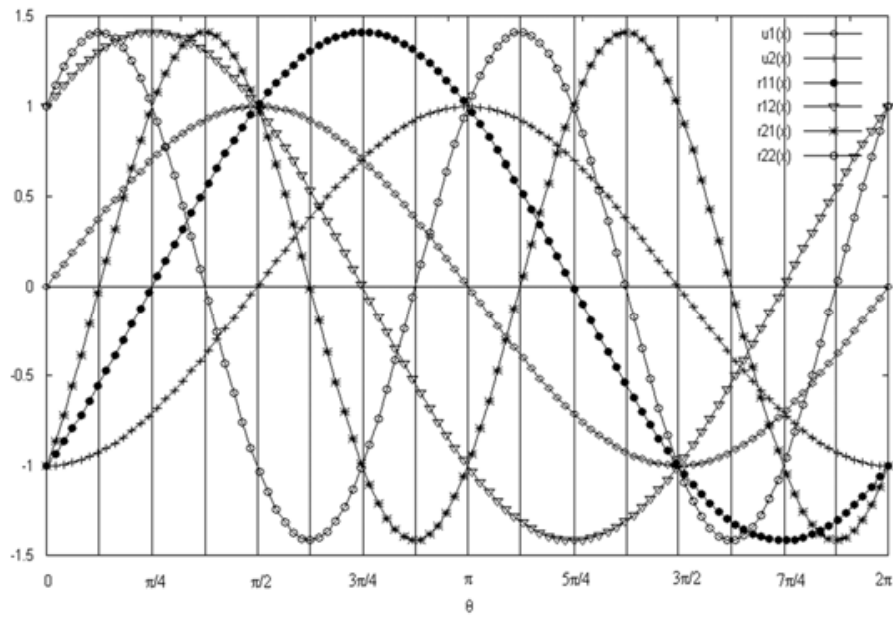

Fig. 9. Backward orthogonal signals and reference signals for $\lambda / 16$ subdivision

From Euler's formula:

$$
e^{j \theta}=\cos \theta+j \sin \theta,
$$

the following equation is obtained:

$$
e^{j n \theta}=\cos n \theta+j \sin n \theta=(\cos \theta+j \sin \theta)^{n} .
$$

Then Eq. 23 is expanded as follows: 


$$
\begin{aligned}
\cos n \theta+j \sin n \theta & =\left(\begin{array}{l}
n \\
0
\end{array}\right) \cos ^{n} \theta+j\left(\begin{array}{l}
n \\
1
\end{array}\right) \cos ^{n-1} \theta \sin \theta+j^{2}\left(\begin{array}{l}
n \\
2
\end{array}\right) \cos ^{n-2} \theta \sin ^{2} \theta+\ldots+j^{n}\left(\begin{array}{l}
n \\
n
\end{array}\right) \sin ^{n} \theta \\
& =\left\{\begin{array}{l}
\left(\begin{array}{l}
n \\
0
\end{array}\right) \cos ^{n} \theta+j\left(\begin{array}{l}
n \\
1
\end{array}\right) \cos ^{n-1} \theta \sin \theta-\ldots+\left(\begin{array}{l}
n \\
n
\end{array}\right) \sin ^{n} \theta, n=4 N \\
\left(\begin{array}{l}
n \\
0
\end{array}\right) \cos ^{n} \theta+j\left(\begin{array}{l}
n \\
1
\end{array}\right) \cos ^{n-1} \theta \sin \theta-\ldots+j\left(\begin{array}{l}
n \\
n
\end{array}\right) \sin ^{n} \theta, n=4 N+1 \\
\left(\begin{array}{l}
n \\
0
\end{array}\right) \cos ^{n} \theta+j\left(\begin{array}{l}
n \\
1
\end{array}\right) \cos ^{n-1} \theta \sin \theta-\ldots-\left(\begin{array}{l}
n \\
n
\end{array}\right) \sin ^{n} \theta, n=4 N+2 \\
\left(\begin{array}{l}
n \\
0
\end{array}\right) \cos ^{n} \theta+j\left(\begin{array}{l}
n \\
1
\end{array}\right) \cos ^{n-1} \theta \sin \theta-\ldots-j\left(\begin{array}{l}
n \\
n
\end{array}\right) \sin ^{n} \theta, n=4 N+3
\end{array}\right.
\end{aligned}
$$

where $N$ is integer and $N \geq 0$.

From Eqs.24, the following equations are obtained:

$$
\begin{gathered}
\left\{\begin{array}{l}
\sin 2 \theta=2 \sin \theta \cos \theta \\
\cos 2 \theta=\cos ^{2} \theta-\sin ^{2} \theta
\end{array}\right. \\
\left\{\begin{array}{l}
\sin n \theta=\left(\begin{array}{l}
n \\
1
\end{array}\right) \cos ^{n-1} \theta \sin \theta-\left(\begin{array}{l}
n \\
3
\end{array}\right) \cos ^{n-3} \theta \sin ^{3} \theta+\ldots-\left(\begin{array}{c}
n \\
n-1
\end{array}\right) \cos \theta \sin ^{n-1} \theta, n=4 N, \\
\cos n \theta=\cos ^{n} \theta-\left(\begin{array}{l}
n \\
2
\end{array}\right) \cos ^{n-2} \theta \sin ^{2} \theta+\ldots+\sin ^{n} \theta
\end{array}, n,\right.
\end{gathered}
$$

where $N$ is integer and $N \geq 1$.

So, from Eqs.16, Eqs.25 and Eqs.26, the following equations are obtained:

$$
\begin{gathered}
\left\{\begin{array}{l}
r_{11}=U_{0} \sin \theta+U_{0} \cos \theta \\
r_{12}=U_{0} \sin \theta-U_{0} \cos \theta^{\prime}
\end{array},\right. \\
\left\{\begin{array}{l}
r_{21}=U_{0}[2 \cos \theta(\sin \theta+\cos \theta)-1] \\
r_{22}=U_{0}[2 \sin \theta(\sin \theta+\cos \theta)-1]
\end{array}, n=2,\right. \\
\left\{\begin{array}{l}
r_{n 1}=U_{0}\left(\cos ^{2^{n-1}} \theta+\left(\begin{array}{c}
2^{n-1} \\
1
\end{array}\right) \cos ^{2^{n-1}-1} \theta \sin \theta-\left(\begin{array}{c}
2^{n-1} \\
2
\end{array}\right) \cos ^{2^{n-1}-2} \theta \sin ^{2} \theta-\ldots+\sin ^{2^{n-1}} \theta\right) \\
r_{n 2}=U_{0}\left(-\cos ^{2^{n-1}} \theta+\left(\begin{array}{c}
2^{n-1} \\
1
\end{array}\right) \cos ^{2^{n-1}-1} \theta \sin \theta+\left(\begin{array}{c}
2^{n-1} \\
2
\end{array}\right) \cos ^{2^{2^{n-1}}-2} \theta \sin ^{2} \theta-\ldots-\sin ^{2^{n-1}} \theta\right)^{\prime} n>2
\end{array}\right.
\end{gathered}
$$

where $n$ is integer. 
The same as the $\lambda / 8$ subdivision and the $\lambda / 16$ subdivision, $u_{1}$ and $u_{2}$ are used to express $r_{n 1}$ and $r_{n 2}$ :

$$
\begin{gathered}
\left\{\begin{array}{l}
r_{11}=u_{1}+u_{2} \\
r_{12}=u_{1}-u_{2}
\end{array}, n=1,\right. \\
\left\{\begin{array}{l}
r_{21}=\frac{2 u_{2}}{U_{0}}\left(u_{1}+u_{2}\right)-U_{0} \\
r_{22}=\frac{2 u_{1}}{U_{0}}\left(u_{1}+u_{2}\right)-U_{0}
\end{array}, n=2,\right. \\
\left\{\begin{array}{l}
r_{n 1}=\frac{1}{U_{0}^{2^{n-1}-1}}\left(u_{2}^{2^{n-1}}+\left(\begin{array}{c}
2^{n-1} \\
1
\end{array}\right) u_{2}^{2^{n-1}-1} u_{1}-\left(\begin{array}{c}
2^{n-1} \\
2
\end{array}\right) u_{2}^{2^{n-1}-2} u_{1}^{2}-\ldots+u_{1}^{2^{n-1}}\right) \\
r_{n 2}=\frac{1}{U_{0}^{2^{n-1}-1}}\left(-u_{2}^{2^{n-1}}+\left(\begin{array}{c}
2^{n-1} \\
1
\end{array}\right) u_{2}^{2^{n-1}-1} u_{1}+\left(\begin{array}{c}
2^{n-1} \\
2
\end{array}\right) u_{2}^{2^{n-1}-2} u_{1}^{2}-\ldots-u_{1}^{2^{n-1}}\right)^{\prime}, n>2,
\end{array}\right.
\end{gathered}
$$

where $n$ is integer.

For $n=1$, the number of subdivision times is 8 .

For $n=2$, the number of subdivision times is 16. This is just shown in Fig.9 during the time of the forward counting and Fig.10 during the time of the backward counting.

Similarly, the $\lambda / 32, \lambda / 64, \lambda / 128, \ldots, \lambda / 2^{n+2}$ subdivision can be obtained where $n$ is integer and $n>2$.

Consequently, a conclusion is obtained as follows:

On the basis of the $\lambda / 2^{n+1}$ bidirectional subdivision, the $\lambda / 2^{n+2}$ bidirectional subdivision of the orthogonal signals can be realized by adding the two reference signals $r_{n 1}$ and $r_{n 2}$ and their zero-cross detection. The zero-cross detection uses the original orthogonal signals and the reference signals $r_{11}, r_{12}, r_{21}, r_{22}, \ldots, r_{(n-1) 1}$ and $r_{(n-1) 2}$ as the positive-negative judgment items for the forward counting and the backward counting.

\section{Error detection and elimination}

In the ideal conditions, the phase difference of the orthogonal signals is $\pi / 2$. However, because of the imperfect design and manufacture of measurement system, the environmental disturbance and the system noise, the signals often have some errors, such as the nonorthogonality, the non-equality of amplitude, the drift of DC signals, and so on (Heydemann, 1981), which have very serious influence on the precision of the fringe subdivision. The typical orthogonal signals with errors are shown as follows:

$$
\left\{\begin{array}{l}
u_{1}^{d}=u_{1}+p \\
u_{2}^{d}=\frac{1}{r}\left(u_{2} \cos a-u_{1} \sin a\right)+q^{\prime}
\end{array}\right.
$$


where $p, q$ are drift of DC, $r$ is non-equality of amplitude coefficient, $a$ is nonorthogonal error, respectively.

(Heydemann, 1981) proposes a universal method to compensate the errors. From Eqs.33, another equation is obtained as follows:

$$
\left(u_{1}+p\right)^{2}+\left(\frac{1}{r} u_{2} \cos a-\frac{1}{r} u_{1} \sin a+q\right)^{2}=U_{0}^{2}
$$

and

$$
\left(u_{1}^{d}-p\right)^{2}+\left[\frac{\left(u_{2}^{d}-q\right) r+\left(u_{1}^{d}-p\right) \sin a}{\cos a}\right]^{2}=U_{0}{ }^{2} .
$$

From Eq.35, the error factors are obtained as follows:

$$
A u_{1}^{d 2}+B u_{2}^{d 2}+C u_{1}^{d} u_{2}^{d}+D u_{1}^{d}+E u_{2}^{d}=1
$$

where

$$
\begin{aligned}
& A=\left(R^{2} \cos ^{2} a-p^{2}-r^{2} q^{2}-2 r p q \sin a\right)^{-1} \\
& B=A r^{2} \\
& C=2 A r \sin a \\
& D=-2 A(p+r q \sin a) \\
& E=-2 A r(r q+p \sin a)
\end{aligned}
$$

respectively. Obviously this is an elliptic function express. The parameter $A, B, C, D$, and $E$ are called the error factors. Therefore, if the error factors $A, B, C, D$, and $E$ are obtained, $p, q$, $r, a$ are calculated as follows:

$$
\left\{\begin{array}{l}
a=\arcsin C(4 A B)^{-\frac{1}{2}} \\
r=\left(\frac{B}{A}\right)^{\frac{1}{2}} \\
p=\frac{2 B D-E C}{C^{2}-4 A B} \\
q=\frac{2 A E-D C}{C^{2}-4 A B}
\end{array} .\right.
$$

From Eqs.37, $u_{1}$ and $u_{2}$ are obtained as follows:

$$
\left\{\begin{array}{l}
u_{1}=u_{1}^{d}-p \\
u_{2}=\frac{1}{\cos a}\left[\left(u_{1}^{d}-p\right) \sin a+r\left(u_{2}^{d}-q\right)\right]
\end{array}\right.
$$


(Heydemann, 1981) sets up a complete method for the error compensation. This method needs at least a period of signals for the calculation of the error factors. Therefore it has the poor real-time performance. (Zumberge et al., 2004, Eom et al., 2001) also calculate error factors for the compensation by least-squares fitting. (Song et al., 2000) uses phasemodulated grating to compensate for nonorthogonal errors. (Keem et al., 2004) uses Jones matrix calculation to investigate the remaining error of a homodyne interferometer with a quadrature detector system. (Cheng et al., 2009) uses the normalized waveforms to eliminate DC offsets and amplitude variation. (Hu \& Zhang, 2011) proposes a method for detection and elimination of the nonorthogonal errors based on digital sampled signals as follows when (Hu et al., 2009, Hu \& Zhang, 2012) are applied in the fringe subdivision.

In (Birch, 1990), when the measured object moves forward, the two orthogonal signals which only contain nonorthogonality error are shown as follows:

$$
\left\{\begin{array}{l}
u_{1}=U_{0} \sin \theta \\
u_{2 f}=U_{0} \sin \left(\theta+\frac{\pi}{2}+\Delta \varphi\right)
\end{array},\right.
$$

where $\Delta \varphi$ is the nonorthogonality error. Similarly, when the measured object moves backward, the original signals which only contain the nonorthogonality error are shown as follows:

$$
\left\{\begin{array}{l}
u_{1}=U_{0} \sin \theta \\
u_{2 b}=U_{0} \sin \left(\theta-\frac{\pi}{2}-\Delta \varphi\right)
\end{array} .\right.
$$

The detection method of $\Delta \varphi$ is as follows:

At first, a counter is defined as $C$ that records the sample number of the original signal $u_{2}$. Then, in the forward movement of the measured object, when the original signal $u_{1}$ crosses zero from negative to positive, the counter $C$ resets itself and begins to count. When the original signal $u_{2}$ crosses zero, the value of the counter $C_{e}$ is saved, and the counter $C$ goes on counting. When the original signal $\mathrm{u}_{1}$ crosses zero from positive to negative, the value of the counter $C_{h p}$ is saved. Consequently, the nonorthogonality error of two original signals can be calculated as follows:

$$
\Delta \varphi=\frac{\frac{1}{2} C_{h p}-C_{e}}{C_{h p}} \pi .
$$

Similarly, when the measured object moves backward, the counter $C$ resets itself and begins to count. When the original signal $u_{2}$ crosses zero, the value of the counter $C_{e b}$ is saved and the counter $C$ goes on counting. When the original signal $\mathrm{u}_{1}$ crosses zero from positive to negative, the value of the counter $C_{h p}$ is saved. the nonorthogonality error of two original signals is calculated as follows:

$$
\Delta \varphi=\frac{C_{e b}-\frac{1}{2} C_{h p}}{C_{h p}} \pi .
$$


The precision of $\Delta \varphi$ is related with the sample number in the half period obviously. When the sample number in the half period is higher, the precision of $\Delta \varphi$ is higher. Moreover, the multiple-averaging technique can be used based on sampling many times to improve the computational accuracy.

If $d$ is defined as the displacement value when the original signal moves a period, the relationship between the displacement $L$ and the phase $\theta$ is as follows:

$$
L=N d+\frac{\theta}{2 \pi} d, \theta \in[0,2 \pi),
$$

where $N$ is the number of periods when the measured object moves, which can be obtained by the cross-zero detection, and $\theta$ can be acquired by the method of subdivision and the counting. If the nonorthogonality error does not exist, Eq. 43 can be represented by the $\lambda / 8$ bidirectional subdivision from (Hu et al., 2009) as follows:

$$
L=\frac{N_{8} d}{8}+\frac{4 \theta}{\pi} d, \theta \in\left[0, \frac{\pi}{4}\right),
$$

where $N_{8}$ is the number of $1 / 8$ periods when the counting system uses $\lambda / 8$ subdivision algorithm, and

$$
N=\frac{N_{8}}{8}
$$

When the nonorthogonality error exists, the algorithm can not divide a signal period into eight subintervals evenly. Therefore Eq.44 is useless if the nonorthogonality error exists. The relationship of the displacement $L$ and $\theta$ has to be redefined.

$C_{p}$ is defined as the sampling value of the signal in a period and can be obtained by means of the cross-zero detection and the counting for the original signals. When the nonorthogonality error does not exist, Eqs.43 and 44 can be shown as follows:

$$
L=N d+\frac{n}{C_{p}} d, n \in\left(-C_{p}, C_{p}\right)
$$

and

$$
L=\frac{N_{8} d}{8}+\frac{n}{C_{p}} d, n \in\left(-\frac{C_{p}}{8}, \frac{C_{p}}{8}\right),
$$

where $n$ is positive when the measured object moves forward or negative when the measured object moves backward. In the Eq. $46, n$ is the count value of the original signal when the original signal does not pass through a complete period. Similarly, in the Eq.47, $n$ is the count value of the original signal when the original signal does not pass through one eighth periods. 


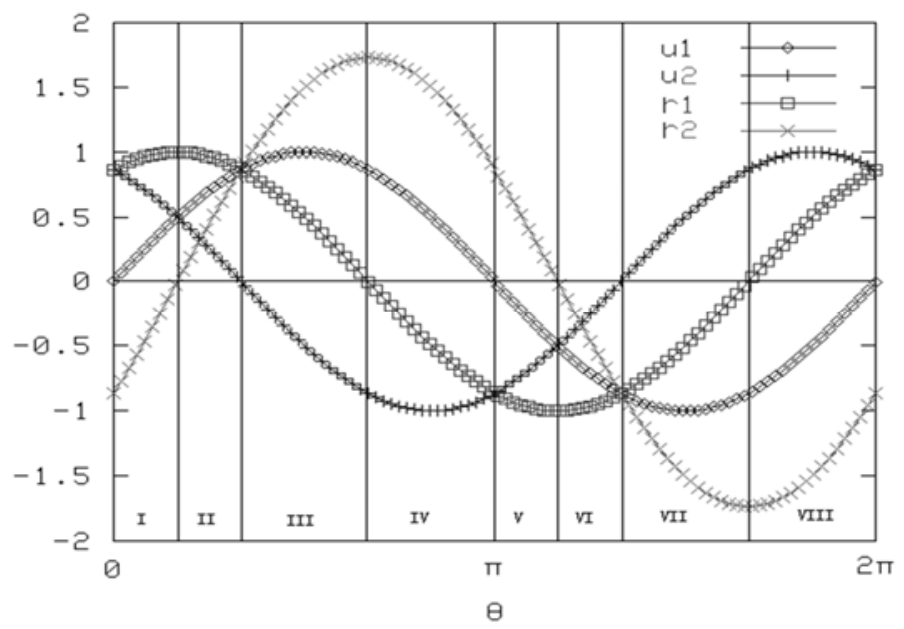

Fig. 10. Subintervals of nonorthogonal signals in $\lambda / 8$ subdivision

When the nonorthogonality error exists, eight subintervals can be arranged in two groups. It is shown as Fig. 12. The first group includes the subinterval I, II, V, and VI, while the second group includes the subinterval III, IV, VII, and VIII. Every subinterval in a same group has the same interval length. So the interval length of eight subintervals can be obtained as Table 1:

\begin{tabular}{ccc}
\hline $\begin{array}{c}\text { Number of } \\
\text { subinterval }\end{array}$ & Range of $\theta$ in $[0,2 \pi)$ & Interval length \\
\hline I & {$\left[0, \frac{\pi}{4}-\frac{\Delta \varphi}{2}\right)$} & $\frac{\pi}{4}-\frac{\Delta \varphi}{2}$ \\
II & {$\left[\frac{\pi}{4}-\frac{\Delta \varphi}{2}, \frac{\pi}{2}-\Delta \varphi\right)$} & $\frac{\pi}{4}-\frac{\Delta \varphi}{2}$ \\
III & {$\left[\frac{\pi}{2}-\Delta \varphi, \frac{3 \pi}{4}-\frac{\Delta \varphi}{2}\right)$} & $\frac{\pi}{4}+\frac{\Delta \varphi}{2}$ \\
IV & {$\left[\frac{3 \pi}{4}-\frac{\Delta \varphi}{2}, \pi\right)$} & $\frac{\pi}{4}+\frac{\Delta \varphi}{2}$ \\
V & {$\left[\pi, \frac{5 \pi}{4}-\frac{\Delta \varphi}{2}\right)$} & $\frac{\pi}{4}-\frac{\Delta \varphi}{2}$ \\
VI & {$\left[\frac{5 \pi}{4}-\frac{\Delta \varphi}{2}, \frac{3 \pi}{2}-\Delta \varphi\right)$} & $\frac{\pi}{4}-\frac{\Delta \varphi}{2}$ \\
VII & {$\left[\frac{3 \pi}{2}-\Delta \varphi, \frac{7 \pi}{4}-\frac{\Delta \varphi}{2}\right)$} & $\frac{\pi}{4}+\frac{\Delta \varphi}{2}$ \\
VIII & {$\left[\frac{7 \pi}{4}-\frac{\Delta \varphi}{2}, 2 \pi\right)$} & $\frac{\pi}{4}+\frac{\Delta \varphi}{2}$ \\
\hline
\end{tabular}

Table 1. The interval length of eight subintervals 
So the displacement $\mathrm{L}$ can be calculated when the nonorthogonality error exists:

$$
L=\left(\frac{\pi-2 \Delta \varphi}{8 \pi} N_{8 a}+\frac{\pi+2 \Delta \varphi}{8 \pi} N_{8 b}\right) d+\frac{n}{C_{p}} d,
$$

where $N_{8 a}$ is the count value of the subinterval when the original signal passes by the subinterval I, II, V, and VI, $\mathrm{N}_{8 b}$ is the count value of the subinterval when the original signal passes by the subinterval III, IV, VII, and VIII, and $n$ is the count value of the original signal when the original signal does not pass through a subinterval. $N_{8 a}, N_{8 b}$, and $\mathrm{n}$ are positive when the measurement platform moves forward or negative when the measurement platform moves backward.

Therefore, without the extra compensation for the nonorthogonality error, the displacement $L$ can be also calculated and the influence of the nonorthogonality error can be eliminated by means of Eq.48.

\section{Implementation platform}

With the development of electronic and computer technologies, many platforms are widely used in the signal processing for orthogonal signals, such as the hardware (Birch, 1990, Downs \& Birch, 1983), FPGA (Hu et al., 2009), the software programming (Zhang et al., 1994, Su et al., 2000), LabVIEW (Yacoot et al., 2001), DSP(Zumberge et al., 2004), and so on. Besides the analog signal processing and the digital signal processing, the image processing is also an important method to realize the fringe analysis (Takeda et al., 1982, Qian, 2004). The image processing system often get data from the CMOS or CCD cameras, and use digital image processing methods to calculate the phase shift. The image processing technique offers many new solutions for the fringe analysis (Patorski \& Styk, 2006, Marengo-Rodriguez et al., 2007, Bernini et al., 2009, Larkin et al., 2001, Pokorski \& Patorski, 2010, Zhang, 2011) and extends the application fields of fringe analysis (Geng, 2011, Riphagen et al., 2008).

\section{Summary}

In this chapter the signal processing methods for orthogonal signals are introduced. The orthogonal signals are widely used in the optical grating and the homodyne interferometers. In order to improve the resolution of these optical instruments, some bidirectional subdivision methods are introduced. Besides the subdivision methods, the algorithms for error detection and elimination are also stated for improving the measurement precision. With the development of the hardware technology and the software technology, the precision dimension measurement technology will be further developed and improved on the resolution, the precision, the real-time characteristics, the applicability, and the maneuverability.

\section{References}

JCGM. (2008). International vocabulary of metrology. Available from: <http://www.bipm.org/utils/common/documents/jcgm/JCGM_200_2008.pdf>. 
Cheng, Z. Gao, H. Zhang, Z. Huang, H. \& Zhu, J. (2006). Study of a dual-frequency laser interferometer with unique optical subdivision techniques. Appl. Opt., Vol. 45, pp. 2246-2250.

Keem, T. Gonda, S. Misumi, I. Huang, Q. \& Kurosawa T. (2004). Removing Nonlinearity of a Homodyne Interferometer by Adjusting the Gains of its Quadrature Detector Systems. Appl. Opt., vol. 43, pp. 2443-2448.

Yoshizawa, T. (2008). Handbook of Optical Metrology - Principles and Applications, CRC Press.

Chu, X. Lü, H. \& Cao, J. (2003). Research on direction recognizing and subdividing method for Moiré(interference) fringes. Chin. Opt. Lett., vol. 1, pp. 692-694.

Cui, J. Li, H. \& Chen, Q. (2000). New digital subdividing snd rester-sensing technique for moiré fringe of grating. Opt. Tech., vol. 26, pp. 591-593.

Chen, B. Luo, J. \& Li, D. (2005). Code counting of optical fringes: methodology and realization. Appl. Opt., vol. 44, pp. 217-222.

$\mathrm{Hu}, \mathrm{H}$. Qiu, X. Wang, J. Ju, A. \& Zhang, Y. (2009). Subdivision and direction recognition of $\lambda / 16$ of orthogonal fringes for nanometric measurement. Appl. Opt., vol. 48, pp. 6479-6484.

Hu, H. \& Zhang, F. (2012). Advanced bidirectional subdivision algorithm for orthogonal fringes in precision optical measurement instruments. Optik, in press. Available from <http:/ / www.sciencedirect.com/science/article/pii/S0030402611005973>.

Heydemann, P. L. M. (1981). Determination and correction of quadrature fringe measurement errors in interferometers. Appl. Opt., vol. 20, pp. 3382-3384.

Zumberge, M. A. Berger, J. Dzieciuch, M. A. \& Parker R. L. (2004). Resolving quadrature dringes in real time. Appl. Opt., vol. 43, pp. 771-775.

Eom, T. B. Kim, J. Y. \& Jeong, K. (2001). The dynamic compensation of nonlinearity in a homodyne laser interferometer. Meas. Sci. and Technol., vol. 12, pp. 1734-1738.

Song, J. H. Kim, K. C. \& Kim, S. H. (2000). Reducing tilt errors in moiré linear encoders using phase-modulated grating. Rev. Sci. Instrum., vol. 71, pp. 2296-2300.

Cheng, F. Fei, Y. T. \& Fan, G. Z. (2009). New method on real-time signal correction and subdivision for grating-based nanometrology. Proc. SPIE., vol. 7284, 728403.

Birch, K. P. (1990). Optical fringe subdivision with nanometric accuracy. Precis. Eng., vol. 12, pp.195-198.

Downs, M. J. \& Birch, K. P. (1983). Bi-directional fringe counting interference refractometer. Precis. Eng., vol. 5, pp. 105-110.

Zhang, G. X. Wang, C. H. \& Li, Z. (1994). Improving the accuracy of angle measurement system with optical grating. CIRP Ann. Manuf. Technol., vol. 43, pp. 457-460.

Su, S. Lü, H. Zhou, W. \& Wang, G. (2000). A software solution to counting and subdivision of Moiré fringe with wide dynamic range. Proc. SPIE, vol.4222, pp. 308-312.

Yacoot, A. Kuetgens, U. Koenders, L. \& Weimann, T. (2001). A combined scanning tunnelling microscope and x-ray interferometer. Meas. Sci. Technol., vol. 12, pp. 1660.

Takeda, M. Ina, H. \& Kobayashi, S. (1982). Fourier-transform method of fringe-pattern analysis for computer-based topography and interferometry. J. Opt. Soc. Am., vol. 72, pp. 156-160.

Qian, K. (2004).Windowed Fourier Transform for Fringe Pattern Analysis. Appl. Opt., vol. 43, pp. 2695-2702. 
Patorski, K \& Styk, A. (2006). Interferogram intensity modulation calculations using temporal phase shifting: error analysis. Opt. Eng., vol. 45, 085602.

Marengo-Rodriguez, F. A. Federico, A. \& Kaufmann, G. H. (2007). Phase measurement improvement in temporal speckle pattern interferometry using empirical mode decomposition. Opt. Commun., vol. 275, pp. 38-41.

Bernini, M. B. Federico, A. \& Kaufmann, G. H. (2009). Normalization of fringe patterns using the bidimensional empirical mode decomposition and the Hilbert transform. Appl. Opt., vol. 48, pp. 6862-6869.

Larkin, K. G. Bone, D. J. \& Oldfield, M. A. (2001). Natural demodulation of two-dimensional fringe patterns. I. General background of the spiral phase quadrature transform. J. Opt. Soc. Am., vol. 18, pp.1862-1870.

Pokorski, K. \& Patorski, K. (2010). Visualization of additive-type moiré and time-average fringe patterns using the continuous wavelet transform. Appl. Opt., vol. 49, pp. 3640-3651.

Zhang, S. (2011). High-resolution 3-D profilometry with binary phase-shifting methods. Appl. Opt., 50(12), 1753-1757.

Geng, J. (2011). Structured-light 3D surface imaging: a tutorial. Adv. Opt. Photon., vol. 3, pp. 128-160.

Riphagen, J. M. van Neck, J. W. van Adrichem, \& L. N. A. (2008). 3D surface imaging in medicine: a review of working principles and implications for imaging the unsedated child. J. Craniofac. Surg., pp. 19, vol. 517-524.

$\mathrm{Hu}, \mathrm{H}$. \& Zhang, F. (2011). Real-time detection and elimination of nonorthogonality error in interference fringe processing. Appl. Opt., vol. 50, pp. 2140-2144. 
(C) 2012 The Author(s). Licensee IntechOpen. This is an open access article distributed under the terms of the Creative Commons Attribution 3.0 License, which permits unrestricted use, distribution, and reproduction in any medium, provided the original work is properly cited. 